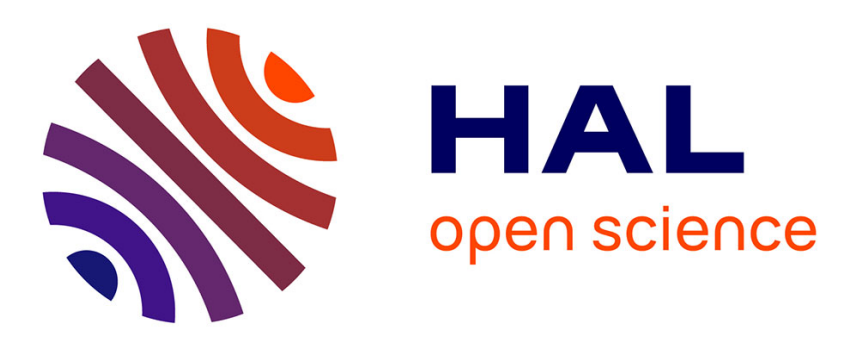

\title{
Numerical studies on Kerr comb generation in Si3N4 resonators with frequency dependent access coupler properties
}

Napoléon Gutierrez, Arnaud Fernandez, Olivier Llopis, Stéphane Calvez, Stéphane Balac

\section{To cite this version:}

Napoléon Gutierrez, Arnaud Fernandez, Olivier Llopis, Stéphane Calvez, Stéphane Balac. Numerical studies on Kerr comb generation in Si3N4 resonators with frequency dependent access coupler properties. SPIE Photonics West, Feb 2019, San-Francisco, United States. hal-02020561

\section{HAL Id: hal-02020561 https://hal.science/hal-02020561}

Submitted on 15 Feb 2019

HAL is a multi-disciplinary open access archive for the deposit and dissemination of scientific research documents, whether they are published or not. The documents may come from teaching and research institutions in France or abroad, or from public or private research centers.
L'archive ouverte pluridisciplinaire HAL, est destinée au dépôt et à la diffusion de documents scientifiques de niveau recherche, publiés ou non, émanant des établissements d'enseignement et de recherche français ou étrangers, des laboratoires publics ou privés. 


\title{
Numerical studies on Kerr comb generation in $\mathrm{Si}_{3} \mathrm{~N}_{4}$ resonators with frequency dependent access coupler properties
}

\author{
N. Gutierrez ${ }^{1}$, A. Fernandez ${ }^{1}$, O. Llopis ${ }^{1}$, S. Calvez ${ }^{1}$, S. Balac ${ }^{2}$ \\ ${ }^{1}$ LAAS-CNRS, University of Toulouse, CNRS, UPS, Toulouse, France \\ ${ }^{2}$ IRMAR, Université of Rennes 1, CNRS, Campus de Beaulieu, 35042 Rennes, France
}

\begin{abstract}
Numerical studies on Kerr frequency comb generation with vertically-coupled whispering-gallery-mode (WGM) $\mathrm{Si}_{3} \mathrm{~N}_{4}$ resonators are presented. These resonators include a frequency-dependent access coupler and are characterized by a free spectral range (FSR) of $220 \mathrm{GHz}$. We present numerical simulations based on the Ikeda map that allows implementation of complex-valued frequency-dependent and non-reciprocal access coupler transfer matrix in the simulation of Kerr comb in the cavities modelled by Arlotti et al ${ }^{1}$. We use a Runge-Kutta 4 Interaction picture (RK4IP) method with adaptive step-size control as developed by $\mathrm{Balac}^{2} \mathrm{el}^{2}$ to circumvent the numerical burden added by this modelling approach and successfully simulate Kerr comb generation using an approach that accurately models any optical cavity that can be considered as spatially one-dimensional regardless of its quality factor, finesse or dispersive properties which comes in useful in this study when access coupling properties degrade the resonator quality factor.
\end{abstract}

Keywords: Racetrack Resonator, Frequency-dependent coupling, Kerr Comb,

\section{INTRODUCTION}

Kerr Frequency Comb (KFC) generation is the process by which an optical spectrum consisting of evenly spaced lines are generated by taking advantage of the Kerr effect. Optical resonators are attractive platforms for KFC generation because of their resonant nature i.e. because of their mode selectivity that and their power enhancement properties. In continuous wave (CW) pumped micro resonators, KFC generation arises in the anomalous dispersion regime when the parametric gain engendered by light circulating inside the cavity overcomes losses and the first modulation sidebands are generated by degenerate Four Wave Mixing (FWM). As more power is accumulated inside the cavity, the generated combs lines interact with each other by nondegenerate FWM forming an evenly space comb with a multiple-FSR spacing ${ }^{3,4}$. As these comb lines become strong enough to create parametric sidebands strong enough to overcome total cavity losses, they broaden occupying the resonator modes between them and merge together creating a FSR-spaced frequency comb ${ }^{3}$.

The power threshold for KFC generation in optical resonators can be optimized by maintaining adequate coupling properties for optimum power enhancement over a large bandwidth. Furthermore, total roundtrip losses can also be optimized so that the losses that have to be overcome by parametric gain in order for FWM to occur are further reduced. Hence, this is why the work of Arlotti et al. on vertically coupled whispering-gallery-mode (WGM) $\mathrm{Si}_{3} \mathrm{~N}_{4}$ resonators design for wideband critical coupling, which has demonstrated coupling geometries that allow critical bandwidths larger than $330 \mathrm{~nm}^{1}$, is so important. These results prove very promising in the context of Kerr frequency comb generation in these platforms as access coupling properties are a crucial factor in a resonator's power enhancement properties and finesse ${ }^{5}$. Furthermore, $\mathrm{Si}_{3} \mathrm{~N}_{4}$ is a material that proves interesting in the context of KFC generation due to its high nonlinear refractive index $\left(\mathrm{n}_{2}=2.510^{-15} \mathrm{~cm}^{2}\right.$. W $\left.{ }^{-1}\right)$, its compatibility with CMOS technologies and the absence of two-photon absorption in telecommunication band ${ }^{6}$. However, as optimized as the access coupling can be, these structures can present spectral regions where the resonator's quality (Q) factor (and thus finesse) is strongly degraded. Moreover, the resonator's access coupler dispersion has a strong influence on the overall resonator dispersion. All this creates problems in numerically simulating Kerr comb generation with the Lugiato-Lefever Equation (LLE) ${ }^{7}$.

This issue can be circumvented by adopting a model that separates intra-cavity dispersion from dispersion in the access coupler such as the Ikeda map ${ }^{8}$, already used in references ${ }^{9,10}$. While this approach is certainly more computationally cost-intensive due to the fact that the step-size has to be inferior to the cavity length, we have put in place a RK4IP method ${ }^{2}$ to reduce computation time and keep computation accuracy high. In addition to this, $\mathrm{Si}_{3} \mathrm{~N}_{4}$ having a higher absorption coefficient than materials such as fluoride glasses $\left(\mathrm{CaF}_{2}\right.$ and $\left.\mathrm{Mg}_{3} \mathrm{~F}_{2}{ }^{11,12}\right)$, 
the quality factor of such resonators doesn't usually exceed $10^{7}$ making numerical simulations of these cavities less time-intensive.

In this paper, we investigate on Kerr comb generation for the three resonators modelled by Arlotti et al. in reference ${ }^{1}$. We start by presenting the modelling approach and parameters used to analyze the frequencydependent cavity transfer properties and Kerr frequency comb generation simulations in section 2. Section 3 describes the transfer properties of the three optical resonators studied in this paper. Section 4 presents simulation results of Kerr comb generation in resonators with frequency-dependent and complex-valued access coupler coefficients.

\section{NUMERICAL MODEL AND PARAMETERS}

Our numerical approach consists in simulating the round-trip to roundtrip evolution of the intra-cavity field of a pumped optical resonator by separating intra-cavity propagation from propagation through the coupler. This is a two time-scale approach that associates a slow time-scale to the variable $t=m t_{R}$ to the $m$-th roundtrip of light circulating inside the resonator with $t_{R}$ the cavity roundtrip time, and a fast time-scale to the variable $\tau$ comprised between $-t_{R} / 2$ and $+t_{R} / 2$ that describes the temporal distribution of the optical signal circulating inside the optical cavity. A schematic of the device modelled is presented in figure 1, along with the absolute value of the coupler's transmission and coupling coefficients at each simulated resonator mode
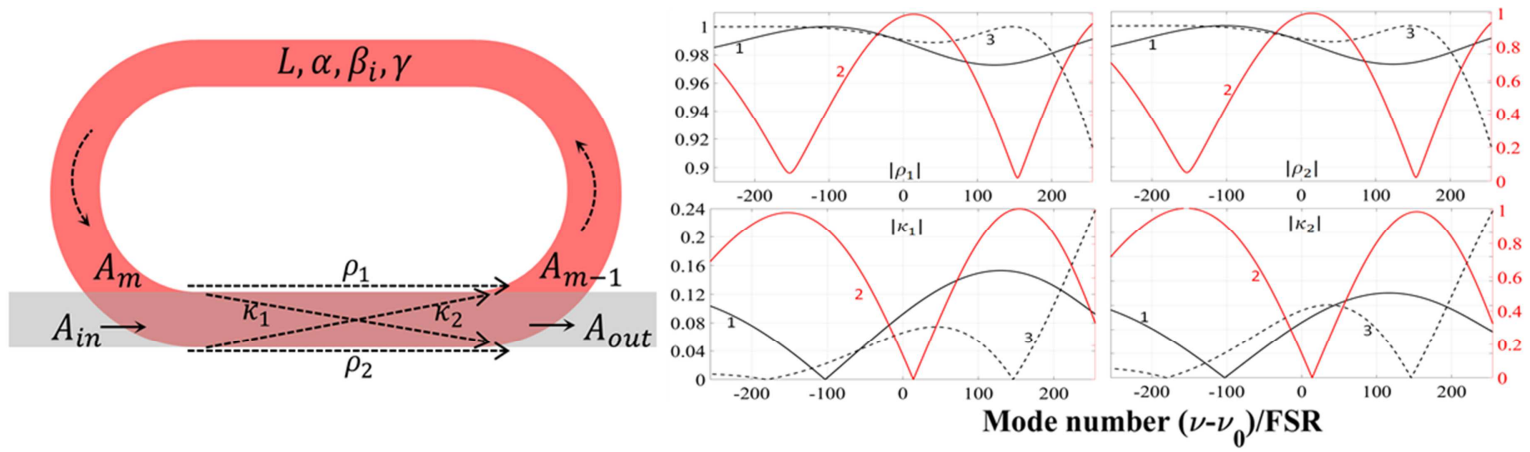

Figure 1. Schematic of the simulated resonator and absolute value of the coupling and transmission coefficients of the 3 access couplers used in simulations. $\rho_{1}, \rho_{2}$ : transmission coefficients. $\kappa_{1}, \kappa_{2}$ : outward and inward coupling coefficients respectively. Coupler 1: Bold black lines. Coupler 2: Bold red lines. Coupler 3: Dashed black lines

In figure $1, A_{i n}$ represents the pump signal at the input of the optical resonator. In our simulation approach, the pump laser is a continuous wave $(\mathrm{CW})$ signal that is superposed to a white noise floor $150 \mathrm{~dB}$ weaker than the pump power. $A_{m}$ represents the intra-cavity signal after propagation through the cavity at the end of roundtrip $m$. $A_{m-1}$ represents the intra-cavity field at round-trip $m$ before propagation through the cavity. Coupler coefficients are represented as a function of mode number which normalizes frequency with respect to the pumped mode and the resonator's FSR. Intra-cavity propagation is described the Non Linear Schrödinger Equation (NLSE) which, limiting dispersion up to the second order, is given by

$$
\frac{\partial \mathrm{A}(\mathrm{z}, \tau)}{\partial z}=-\frac{\alpha}{2} \mathrm{~A}(\mathrm{z}, \tau)-i \frac{\beta_{2}}{2} \frac{\partial^{2} \mathrm{~A}(\mathrm{z}, \tau)}{\partial \tau^{2}}+i \gamma \mathrm{A}(\mathrm{z}, \tau)|\mathrm{A}(\mathrm{z}, \tau)|^{2}
$$

In equation (1), parameter $\beta_{2}$ models dispersion during propagation, parameter $\alpha$ models field attenuation and $\gamma$ is the Kerr coefficient that models the nonlinear Kerr effect during propagation. Material and geometrical properties (e.g. bending radius) of the racetrack resonator have a direct impact on the dispersive properties and propagation losses of the ring cavity ${ }^{13}$. Spectral dependence of $\alpha$ and $\beta_{2}$ depicted in figure 2 shows respectively an attenuation of $29.69\left(\mathrm{~m}^{-1}\right)$ and an anomalous chromatic dispersion of $-18.88\left(\mathrm{ps}^{2} \mathrm{~km}^{-1}\right)$ at $1.55 \mu \mathrm{m}$ (pump laser wavelength). The non-linear index $n_{2}$ combined with mode effective area $A_{\text {eff }}$ lead to a Kerr coefficient $\gamma=1200\left(W^{-1} \cdot \mathrm{km}^{-1}\right)$. 

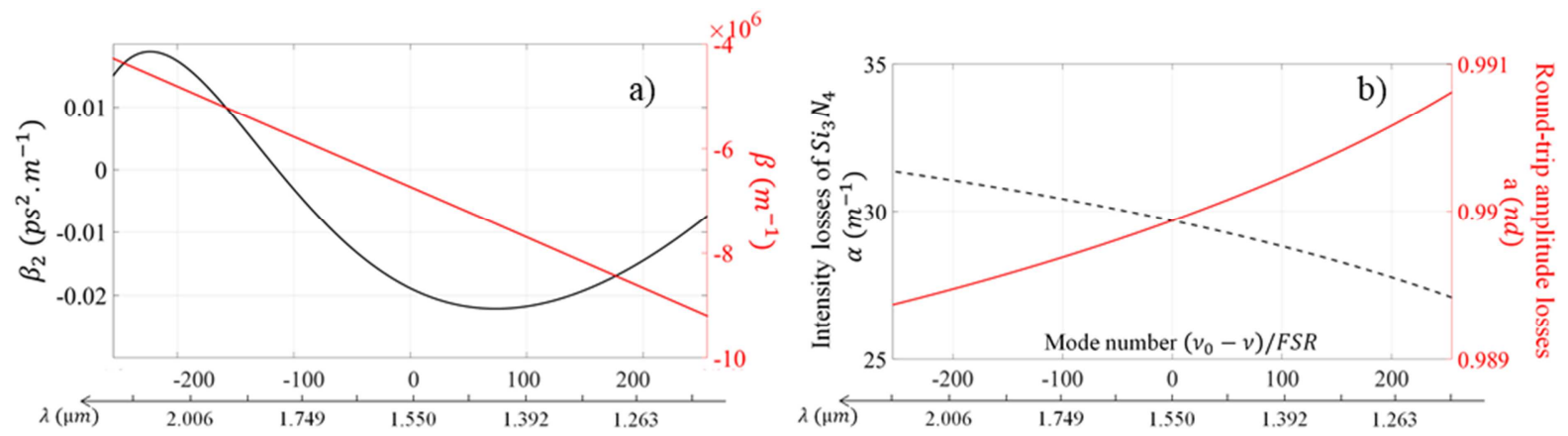

Figure 2. a) Representation of the cavity's second order dispersion parameter and wave vector as functions of mode number. b) Material absorption coefficient and power attenuation per roundtrip as functions of mode number.

Access coupler coefficients are complex valued and frequency dependent. They are used to model not only linear propagation through a coupler of non-zero length but also dispersive effects. In fact, the magnitude values of $\rho_{l}$, $\rho_{2}, \kappa_{1}, \kappa_{2}$ model the amplitude of the transfer between input waveguide and ring cavity while the phase of these coefficients models changes in the optical path of the different spectral components propagating through the coupler, i.e. dispersion during propagation through the coupler. The passage of light through the access coupler is modelled by a transfer matrix that describes signal transfer between the input/output of the resonator and the cavity in the spectral domain.

$$
\left[\begin{array}{c}
\tilde{A}_{m} \\
\tilde{A}_{\text {out }}
\end{array}\right](\lambda)=\left[\begin{array}{cc}
\kappa_{1} & \rho_{1} \\
\rho_{2} & \kappa_{2}
\end{array}\right]\left[\begin{array}{c}
\tilde{A}_{\text {in }} \\
\tilde{A}_{m-1}
\end{array}\right](\lambda)
$$

Propagation through the access coupler is performed numerically at the beginning of every iteration in the spectral domain with the help of fast Fourier transforms (FFT), respecting relation (2). Then, intra-cavity propagation is performed using a RK4IP solver of the NLSE through the length of the cavity taking into account fluctuations of the dispersion and absorption parameters.

This approach has a higher computation cost than ones based in coupled modes equation or split-step simulations of the LLE because it forces the user to adopt a step-size that is lower than the cavity physical length. However, by separating the treatment of intra-cavity propagation and propagation through the access coupler, this two time-scale approach allows for the implementation of non-reciprocal access coupler properties. Furthermore, this approach accurately models Kerr Frequency Comb generation in optical resonator for any optical cavity regardless of its finesse, quality factor or dispersion.

\section{MODELLING OF THE CAVITY TRANSFER PROPERTIES}

\subsection{Transfer function}

A resonator's transfer properties give useful insight about its resonance linewidth, cavity enhancement properties and resonance mode structure. To determine the transfer properties of an optical resonator, it is necessary to consider the ring resonator behavior in linear regime i.e. with very low optical power circulating in the cavity such that the non-linear term doesn't manifest itself in the NLSE (see eq. 1). A very straightforward solution of the propagation equation under this circumstances allows us to determine the frequency dependent $\mathrm{Q}$ factor, free spectral range and power enhancement factor (PEF) of our optical resonator. Indeed, having access to the frequency-dependence of the quantities listed above provides substantial information concerning frequency dependent resonator losses as well as resonance mismatch that subsequently affect KFC generation and reveals the impact on the coupler design. By assuming the absence of Kerr nonlinearity in equation (1), the intra-cavity field propagation admits an analytical solution given by equation (3).

$$
\tilde{A}_{m}\left(v_{k}\right)=\tilde{A}_{m-1}\left(v_{k}\right) e^{-\left(\frac{\alpha}{2}+j\left(2 \pi v_{k}\right)^{2} \frac{\beta_{2}}{2}\right) L}
$$


Here, $v_{k}$ corresponds to the frequency at mode $k$ in an evenly spaced frequency grid $v_{k}=k . F S R(k \in \mathbb{Z})$. By combining eq. (3) with the coupler's transfer function (2), on can easily obtain the resonator's transfer function $T(\varphi)$ in the steady state regime at a given frequency which is given by the relation

$$
T(\varphi)=\frac{\tilde{A}_{\text {out }}}{\tilde{A}_{\text {in }}}=\frac{\rho_{2}+\left(\kappa_{1} \kappa_{2}-\rho_{1} \rho_{2}\right) \hat{L} \widehat{D} e^{j \varphi}}{1-\kappa_{1} \hat{L} \widehat{D} e^{j \varphi}}
$$

In equation (4), the ring attenuation and dispersion per roundtrip are represented respectively through operators $\hat{L}=e^{-\frac{\alpha}{2} L}$ and $\widehat{D}=e^{-j\left(2 \pi v_{k}\right)^{2} \frac{\beta_{2}}{2} L}$. The variable $\varphi$ represents the linear phase accumulated by the intra-cavity field over one round-trip with respect to the FSR spaced frequency grid. This linear phase is linked to a shift in frequency $f \in[-F S R / 2 ; F S R / 2]$ which represents the frequency detuning in the vicinity of the $k^{\text {th }}$ optical mode: $k 2 \pi+\varphi=2 \pi \frac{v_{k}+f}{F S R}$.

\subsection{Quality factor and Power enhancement factor}

The resonator's quality factor $\mathrm{Q}$ is linked to the photon storage time of the optical resonator. In the spectral domain the $\mathrm{Q}$ factor is inversely proportional to the full-width at half maximum $\Delta f_{1 / 2}$ of the cold cavity transfer function squared modulus $|T(\varphi)|^{2}$ at resonance. It is expressed as following: $Q=v_{0} / \Delta f_{1 / 2}$, in which $v_{0}$ represents the absolute frequency at resonance. Experimental spectro-RF metrology technique invoke this $\mathrm{Q}$ factor expression for measurement ${ }^{14}$. In this numerical work, we compute the local Q factor at each resonator mode by referring to equation (4) for each of the three simulated optical resonators. Increasing resonator's Q factor reduces the total cavity losses that have to be overcome by parametric gain in order to start Kerr comb generation and thus reduces the pump power threshold. Another way to decrease the comb generation threshold is to optimize the accumulation of optical power inside an optical cavity at resonance. Pump storage efficiency is quantified by a resonator's power enhancement factor (PEF). It is determined, again, using equations (2) and (3) to determine the ratio of intra-cavity power with respect to pump power. The analytical description of the cavity PEF is given by equation (5)

$$
P E F=\frac{\left|\tilde{A}_{m-1}\right|^{2}}{\left|\tilde{A}_{i n}\right|^{2}}=\frac{\left|\kappa_{1}\right|^{2}}{\left|1-\hat{L} \rho_{1}\right|^{2}}
$$

For a passive ring cavity (which is the case in our study), total cavity losses $\left|\widehat{L} \rho_{1}\right|<1$ and critical coupling only occurs for the singular condition: $\widehat{L}=\rho_{1}$ corresponding to a maximum achievable PEF with a given propagation medium. Since the ring resonator intensity losses $\hat{L}$ and the coupling properties are both frequency dependent, this inevitably degrades the PEF and affects coupling regime. The frequency-dependent quality factor and PEF of the three resonators studied in this letter are depicted in figure 3 .
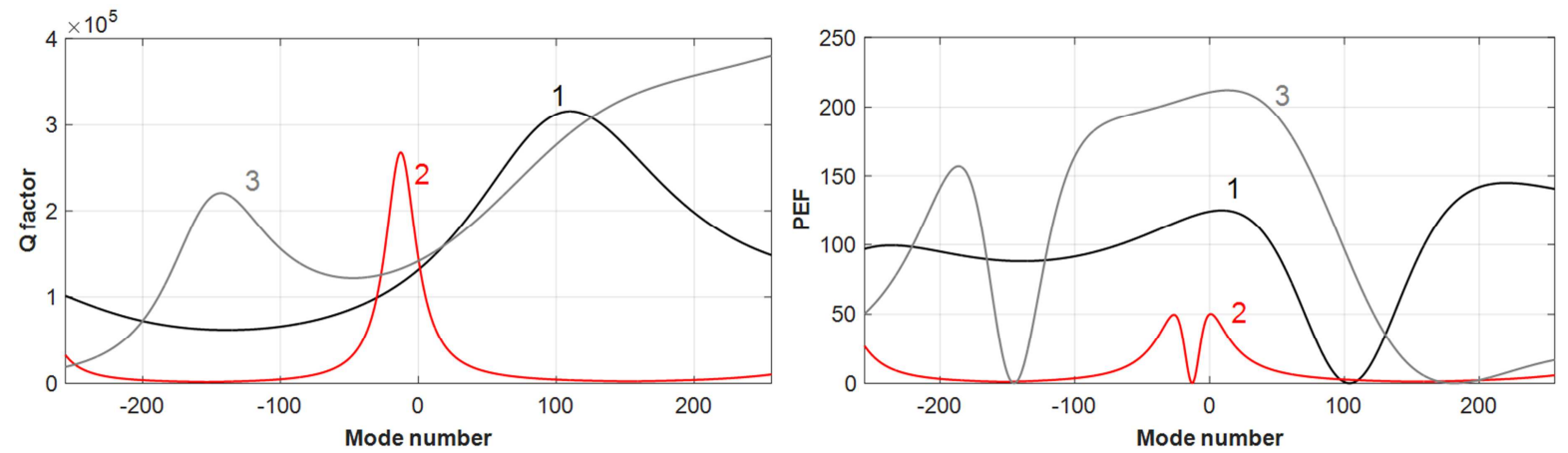

Figure 3. a) Representation of the Quality factor and Power enhancement factor as functions of modes number for the three simulated resonators. Black line: Resonator 1; Red line: Resonator 2; Gray line: Resonator 3.

We observe a similar $\mathrm{Q}$ factor $\approx 1.410^{5}$ at the pumped mode for all three cavities; however the coupling design affects drastically the $\mathrm{Q}$ factor value as mode number increases. It is easy to distinguish the case corresponding to Coupler 2 as it corresponds to a high quality factor over a considerably narrower bandwidth compared to that of the two other cases.

\subsection{Cold resonance mismatch: Intra-cavity and coupler dispersion}

Another critical aspect in Kerr comb generation is total roundtrip dispersion. As stated in section 1, KFC generation in optical resonators arises from the compensation of dispersive effects by nonlinear effects. The fact that the optical path of light is frequency dependent produces changes in the spectral position of each resonance of an optical resonator. If dispersion is adequate, this allows the Kerr effect to pull the resonator modes into an evenly spaced frequency-grid as pump power is accumulated inside the cavity. In order for modulation instability to occur and give rise to an optical frequency comb, the mode structure of the cavity has to present a concave shape at the pump wavelength. This means having a second order dispersion parameter that is negative at the pump wavelength. There are two sources of dispersion in the model presented in 
this paper and those are intra-cavity dispersion modeled by $\beta_{2}$ coefficient in the propagation equation and the phase of the complex-valued coupler coefficients. The phase modulation term $\left(2 \pi v_{k}\right)^{2} \beta_{2} / 2$ in equation (3) models the resonance frequency mismatch due to intra-cavity dispersion of a resonator and can be used to obtain its contribution to the cold cavity resonance mismatch of a resonator with the equation

$$
\Delta v_{c}=\left(\sum_{n>1} \frac{\beta_{n}}{n !}\left(2 \pi v_{k}\right)^{\mathrm{n}}\right) \frac{F S R}{2 \pi}
$$

Since the contribution of the access coupler to the cold cavity resonance mismatch is modelled by the coupler's transfer matrix coefficients, it is necessary to use equation (4) to obtain the global cold cavity resonance mismatch. Numerically, we find the resonance position of each resonator mode using equation (4) and then compute the difference between resonance position and the evenly spaced frequency grid $v_{k}=k . F S R(k \in \mathbb{Z})$ obtaining the cold resonance frequency mismatch of each resonator.
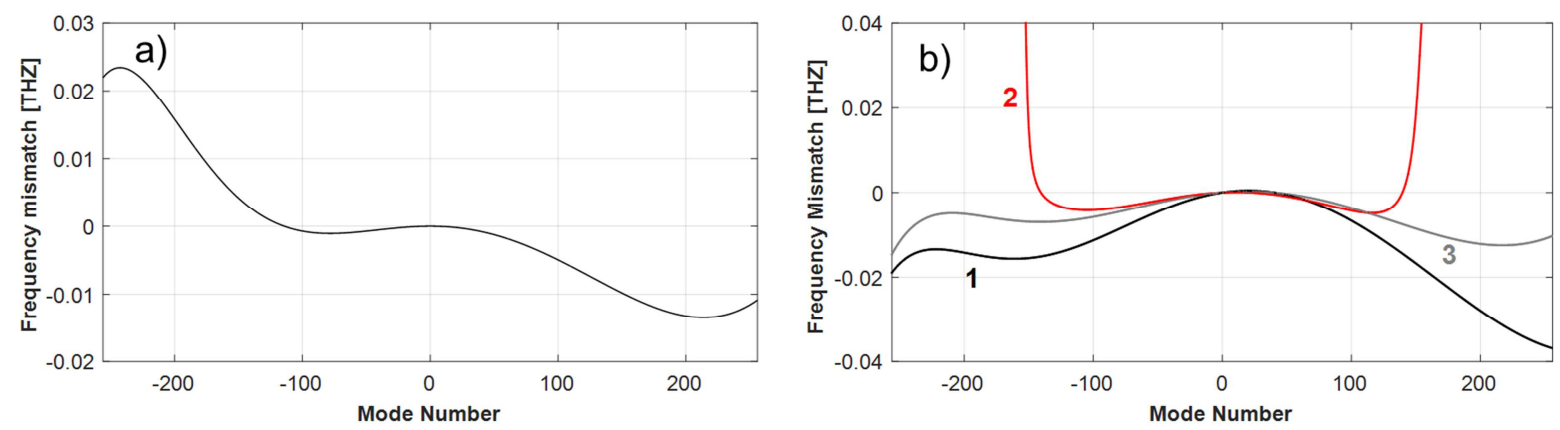

Figure 4. a) Cold cavity resonance mismatch induced by intra-cavity dispersion only (obtained using equation (6)).

b) Cold cavity resonance mismatch induced by total roundtrip dispersion (intra-cavity and coupler) of each simulated resonator.

Figure 4 depicts the cold cavity resonance mismatch computed without taking coupler dispersion into account in figure (a). This effect is mutual to all the simulated optical resonators and is obtained by using equation (6). Figure 4(b) depicts the cold cavity resonance mismatch computed using equation (4) to determine the exact spectral position of each resonator mode with respect to an equally, FSR-spaced frequency grid. Figure 4 shows how in the resonators modelled by Arlotti et al., total round-trip dispersion is strongly affected by coupler geometry. This highlights the importance of taking into account dispersion effects during propagation through the access coupler.

Furthermore, the simple mathematical analysis that was put in place in this section allows us to determine which of the three resonators presents better properties for Kerr comb generation. In terms of frequency-dependent losses, a high quality factor over a large bandwidth is desired so that parametric gain engendered by the pump signal circulating inside the cavity has lower losses to overcome in order for degenerate FWM to occur and give rise to an optical frequency comb. In this category, resonators 1 and 3 are clearly better options than resonator 2 . With respect to the cavity PEF, it is desirable to have the strongest PEF possible at the pumped mode to best take advantage of the power enhancement properties of the cavity and reduce the pump power threshold necessary for Kerr comb generation even further. Concerning resonator dispersion, as long as dispersion is anomalous at the pumped mode, a flatter resonance mismatch curve lends itself to the formation of broader frequency combs ${ }^{15}$. Figure 4 b) shows that resonator 2 possesses the best adapted mismatch curve even suggesting the possibility of dispersive-wave formation around modes -140 and $140 ;{ }^{16}$.

\section{SIMULATION RESULTS}

Kerr comb simulations rely on the model presented in section 2. Because of Kerr nonlinearity, the phase of light propagating in the optical medium is modulated by its own intensity. The term $i \gamma\left|A_{m}\right|^{2} A_{m}$ in the nonlinear Schrödinger equation accounts for the Kerr non-linear phase shift accumulated by light during propagation. This phase shift is due to the modification of the medium's refractive index by the Kerr effect effectively lengthening the optical path of light circulating in the optical resonator as power is increased.

In the context of nonlinear optical cavities, as optical power is accumulated inside the resonator, the pumped mode resonance shifts towards longer wavelengths (lower frequencies) due to self-phase modulation. Because of this, controlling the pump laser frequency detuning with respect to the pumped mode resonance is crucial in practice and in Kerr comb simulations. In order to efficiently accumulate power inside a non-linear optical cavity, the frequency of the pump laser has to be controlled in such a manner that it follows the Kerr-induced resonance shift that takes place as optical power is accumulated inside the 
optical cavity. This means that the pump laser has to be tuned from higher to lower frequencies across the pumped mode resonance in order to follow the resonance drift caused by the Kerr effect as light is accumulated in the cavity. In simulations, the phase detuning of the pump laser with respect to the cold cavity resonance is controlled via a detuning parameter $\delta_{0}$ such that

$$
A_{\text {in }}(\tau)=A(\tau) e^{i \delta_{0}}
$$

With $A(\tau)$ corresponding to the envelope of the pump optical field at round-trip $m$. In simulations we use a CW pump with a white noise floor $150 \mathrm{~dB}$ lower than the pump that serves as a seeding signal for degenerate FWM interactions. The quantity $\delta_{0}$ is the linear phase detuning between the pump signal and the closest cold cavity resonance.

In section 3, the Power enhancement factor of each resonator was established. As this quantity strongly differs at the pumped mode $(1550 \mathrm{~nm})$, pump power used in simulations has been adapted for each resonator so that the quantity $P E F(1550 \mathrm{~nm}) P_{\text {pump }}$ stays constant for all three cases in order to be able to compare the simulation results in a compelling way. In simulations, pump power is adjusted to keep the quantity $P E F(1550 \mathrm{~nm}) P_{\text {pump }}=210 \mathrm{~W}$. This comes to be pumping powers in simulations of $1.7 \mathrm{~W}$ for resonator 1 ; a simulated pump power of $4.2 \mathrm{~W}$ for resonator 2 which has the lowest PEF at the pumped mode and a simulated pump power of $1 \mathrm{~W}$ for resonator 3 . Figure 5 shows the average intra-cavity power during laser scan simulations performed over the cavity resonance for each case of study with normalized pump power as well as the intra-cavity field temporal distribution and the intra-cavity field spectra for the 3 resonators.

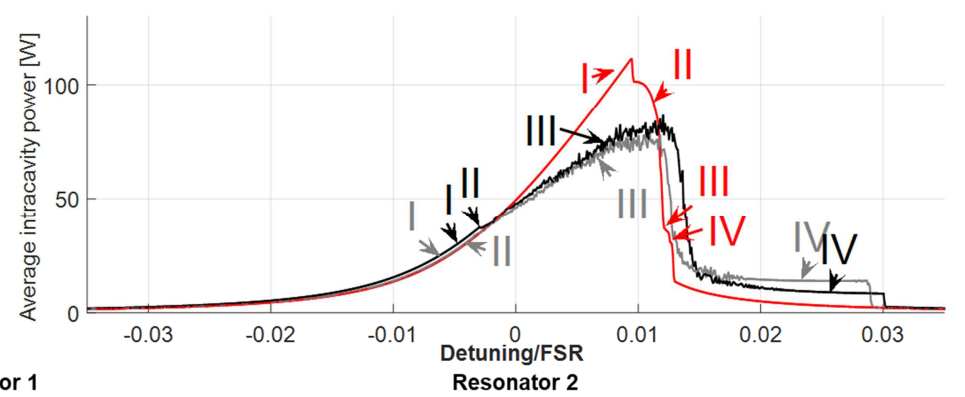

Resonator 1
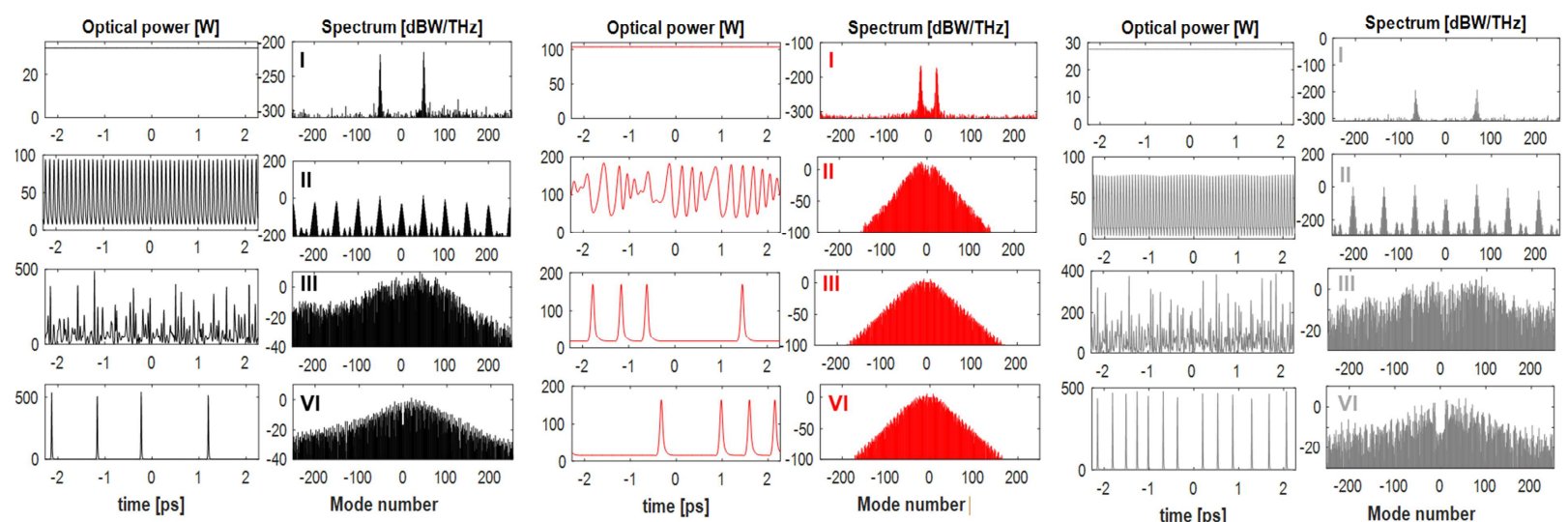

Figure 5. Evolution of intra-cavity field temporal distribution and intra-cavity spectrum during laser scan simulations for each simulated coupler. For resonators 1 and 3: I. Apparition of the first modulation sidebands. II.

Formation of temporal patterns inside the cavity. III. Chaotic comb regime. IV. Multiple pulse regime.

The different functioning regimes in frequency scan simulations can be identified in figure 5. After the apparition of the first modulation sidebands in fig 5.I) comes the formation of temporal patterns (II) as the detuning of the pump laser is performed ${ }^{17}$. These patterns correspond to a phase-locked frequency comb with a comb spacing equal to an integer number of cavity FSRs and are not observed in resonator 2.

As the detuning of the pump laser continues, these generated sidebands widen and overlap filling the resonator modes between them ${ }^{3}$. This leads to a broad but incoherent spectrum with a comb spacing equal to the resonator's free spectral range. The incoherent phase relationship between the spectral components of the comb translates to a chaotic temporal signal as seen for resonators 3 and 1 .

Finally, when the stable multiple pulse regime is attained, a spectrum with a well-defined phase relationship between comb teeth is generated inside the resonator ${ }^{18,19}$. The envelopes of the spectra generated are not smooth due to the fact that multiple pulses circulate inside the resonator at the same time. However, the temporal spacing between pulses remains constant and so the stable spectrum in the pulsed regime results from the superposition of each spectrum associated to each pulse circulating in the cavities. 
Resonator 1 has a wide band high quality factor with a peak around mode -100 and a dip around mode 150. Low total losses allow for the formation of a large comb with circulating $\mathrm{CW}$ power of around $30 \mathrm{~W}$ inside the cavity. Because losses remain of the same order of magnitude over the whole frequency span in simulations, the shape of the intra-cavity spectra generated in this resonator is shaped mainly by total resonator dispersion.

Resonator 2 has a high quality factor over a narrow bandwidth close to the pumped mode that quickly degrades as mode number increases reaching lows of the order of magnitude $10^{3}$ around modes 140 and -140 (fig 3.). A substantially higher $\mathrm{CW}$ power circulating inside the cavity (around $95 \mathrm{~W}$ ) is necessary in order for parametric gain to overcome total cavity losses as seen in figure 5. Furthermore, in the case of this resonator, the generated KFCs are shaped by total resonator losses as there is a very strong contrast of a factor of around 100 between the quality factor at the pumped mode and the lowest quality factor. The cold cavity mode structure of this resonator (fig 4.) even suggest the potentiality of the generation of dispersive waves not far from modes 140 and $-140{ }^{16,20}$ but with losses being most important in these particular regions, this effect does not take place. This resonator is an example of a case where frequency comb bandwidth is limited by the degradation with frequency of total resonator losses.

Resonator 3 presents what seem to be the best KFC generation properties of the 3 resonators with a frequency-dependent Q factor profile that remains highest on average of all three couplers and a flatter cold cavity mismatch curve than resonator 1. The first modulation sidebands are generated with circulating powers of around $30 \mathrm{~W}$ as in resonator 1 due to the fact that both resonators have quality factor spectral profiles that maintain and order of magnitude of at least $10^{5}$. As is the case with resonator number 1 , the spectrum generated with resonator 3 parameters is shaped by the cold cavity mode structure (i.e. total resonator dispersion).

\section{CONCLUSIONS}

In summary, we have presented in this paper the impact that access coupler geometry can have in the wideband transfer function of an optical resonator and we have presented by the means of numerical simulations, the consequences this entails for the generation of Kerr frequency combs.

The modelling approach used in this work predicts that access coupler geometry will have a strong impact in the spectral distribution of the quality factor of the resonator as well as the resonator's mode structure (even more so than intra-cavity dispersion in this particular study). Given that both, losses and cold resonator mode structure, are affected by the coupler geometry, we have run numerical simulations based the Ikeda Map of the resonators in order to study Kerr Frequency comb generation in these structures. Simulations show that the degradation of a resonator's quality factor can strongly hinder the comb generation process and in turn have allowed us to determine that losses can become the limiting factor in comb bandwidth even when dispersion lends itself well for Kerr comb generation.

These results highlight the importance of access coupler design particularly with vertically coupled $\mathrm{Si}_{3} \mathrm{~N}_{4}$ waveguide resonators. Furthermore, simulations predict promising Kerr comb generation results with access couplers 1 and 3 as designed by Arlotti et al an applied to $220 \mathrm{GHz}$ high confinement $\mathrm{Si}_{3} \mathrm{~N}_{4}$ race-track resonators. Resonator 3 particularly predicts the formation of large frequency combs with as low as $1 \mathrm{~W}$ of pump power with a structure that favors CMOSintegration $\left(\mathrm{Si}_{3} \mathrm{~N}_{4}\right)$ and Kerr nonlinearity due to high confinement.

\section{REFERENCES}

[1] C. Arlotti, O. Gauthier-Lafaye, A. Monmayrant, and S. Calvez, "Achromatic critically coupled racetrack resonators," J. Opt. Soc. Am. B, vol. 34, no. 11, p. 2343, Nov. 2017.

[2] S. Balac, A. Fernandez, F. Mahé, F. Méhats, and R. Texier-Picard, "The Interaction Picture method for solving the generalized nonlinear Schrödinger equation in optics," ESAIM Math. Model. Numer. Anal., vol. 50, no. 4, pp. 945-964, Jul. 2016.

[3] T. Herr et al., "Universal formation dynamics and noise of Kerr-frequency combs in microresonators," Nat. Photonics, vol. 6, no. 7, pp. 480-487, Jun. 2012.

[4] T. Herr et al., "Temporal solitons in optical microresonators," Nat. Photonics, vol. 8, no. 2, pp. 145-152, Dec. 2013.

[5] A. Yariv, "Universal relations for coupling of optical power between microresonators and dielectric waveguides," Electron. Lett., vol. 36, no. 4, pp. 321-322, 2000.

[6] J. S. Levy, A. Gondarenko, M. A. Foster, A. C. Turner-Foster, A. L. Gaeta, and M. Lipson, "CMOScompatible multiple-wavelength oscillator for on-chip optical interconnects," Nat. Photonics, vol. 4, no. 1, pp. 37-40, Jan. 2010. 
[7] S. Coen, H. G. Randle, T. Sylvestre, and M. Erkintalo, "Modeling of octave-spanning Kerr frequency combs using a generalized mean-field Lugiato-Lefever model," Opt. Lett., vol. 38, no. 1, p. 37, Jan. 2013.

[8] K. Ikeda, "Multiple-valued stationary state and its instability of the transmitted light by a ring cavity system," Opt. Commun., vol. 30, no. 2, pp. 257-261, Aug. 1979.

[9] T. Hansson and S. Wabnitz, "Frequency comb generation beyond the Lugiato-Lefever equation: multistability and super cavity solitons," J. Opt. Soc. Am. B, vol. 32, no. 7, p. 1259, Jul. 2015.

[10] N. Gutierrez, A. Fernandez, O. Llopis, S. T. Chu, and S. Balac, "An iterative method for the dynamic modeling of ultra-short pulse generation in nonlinear optical ring resonator," 2017, pp. 1-1.

[11] I. S. Grudinin, N. Yu, and L. Maleki, "Generation of optical frequency combs with a CaF 2 resonator," Opt. Lett., vol. 34, no. 7, pp. 878-880, 2009.

[12] W. Liang et al., "High spectral purity Kerr frequency comb radio frequency photonic oscillator," Nat. Commun., vol. 6, p. 7957, Aug. 2015.

[13] D. J. Moss, R. Morandotti, A. L. Gaeta, and M. Lipson, "New CMOS-compatible platforms based on silicon nitride and Hydex for nonlinear optics," Nat. Photonics, vol. 7, no. 8, pp. 597-607, Jul. 2013.

[14] Z. Abdallah, Y. G. Boucher, A. Fernandez, S. Balac, and O. Llopis, "Radio frequency spectral characterization and model parameters extraction of high Q optical resonators," Sci. Rep., vol. 6, Jun. 2016.

[15] S. Coen and M. Erkintalo, "Universal scaling laws of Kerr frequency combs," Opt. Lett., vol. 38, no. 11, pp. 1790-1792, 2013.

[16] C. Bao et al., "High-order dispersion in Kerr comb oscillators," J. Opt. Soc. Am. B, vol. 34, no. 4, p. 715, Apr. 2017.

[17] A. Coillet et al., "Azimuthal Turing Patterns, Bright and Dark Cavity Solitons in Kerr Combs Generated With Whispering-Gallery-Mode Resonators,” IEEE Photonics J., vol. 5, no. 4, pp. 6100409-6100409, Aug. 2013.

[18] H. Guo et al., "Universal dynamics and deterministic switching of dissipative Kerr solitons in optical microresonators," Nat. Phys., vol. 13, no. 1, pp. 94-102, Sep. 2016.

[19] M. Karpov et al., "Universal dynamics and controlled switching of dissipative Kerr solitons in optical microresonators," Nat. Phys., vol. 13, no. 1, pp. 94-102, Sep. 2016.

[20] S. Wang, H. Guo, X. Bai, and X. Zeng, "Broadband Kerr frequency combs and intracavity soliton dynamics influenced by high-order cavity dispersion,” Opt. Lett., vol. 39, no. 10, p. 2880, May 2014. 